\title{
CONTEÚDOS DE BIOLOGIA EM VESTIBULARES SERIADOS
}

\author{
MAÍRA ELIAS MANZANO \\ Mestranda do Programa de Pós-Graduação Interunidades em Ensino de Ciências, \\ do Instituto de Física, da Universidade de São Paulo \\ m.manzano@usp.br
}

SONIA BUENO CARVALHO LOPES

Professora doutora do Departamento de Zoologia, do Instituto de Biociências, da Universidade de São Paulo

sonialop@ib.usp.br

\begin{abstract}
RESUMO
A adoção de novos processos seletivos para o acesso de alunos às universidades só foi possível com a promulgação da Lei de Diretrizes e Bases da Educação Nacional n. 9.394/96. O vestibular seriado é uma dessas propostas alternativas, que prevê uma avaliação sistemática dos alunos ao término de cada ano do ensino médio. O propósito deste estudo foi caracterizar o vestibular seriado, hoje presente em 22 instituições públicas, quanto ao seu objetivo, estilos de avaliação e conteúdos da disciplina de Biologia distribuídos nos módulos referentes às três séries do ensino médio. Averiguamos que os diferentes programas analisados não apresentam uniformidade em diversos aspectos, tais como número de vagas, estrutura de avaliação e conteúdo programático. Verificamos, ainda, a ausência de consenso sobre a sequência a ser adotada para o ensino de Biologia e a resultante restrição para que o aluno possa preparar-se para as provas seriadas em mais de uma instituição.
\end{abstract}

ACESSO AO ENSINO SUPERIOR - CONCURSO VESTIBULAR - BIOLOGIA - ENSINO MÉDIO

\begin{abstract}
BIOLOGY CONTENT IN UNIVERSITY ENTRANCE EXAMS BY PERIODIC ASSESSMENT. The adoption of new student selection processes for university entrance was only made possible by enactment of Education Law no 9,394/96. The university entrance exam by periodic assessment is one of these alternative proposals that provides for a systematic evaluation of the students at the end of each high school year. The purpose of this study was to describe the university entrance exam by periodic assessment, used today used in 22 public institutions, concerning: its objective, assessment styles and the content of the Biology discipline, which is divided into modules that refer to the three grades of high school. We noted that there is no uniformity in the different programs analyzed in several of their aspects, such as the number of places, assessment structure and program content. We also observed a lack of consensus regarding the
\end{abstract}


sequence to be adopted for the teaching of Biology and the resulting restriction for the student being able to be prepared for the periodic tests in more than one institution.

ACESS TO HIGHER EDUCATION - BIOLOGY - SECONDARY EDUCATION - HIGHER EDUCATION

O ingresso ao ensino superior é um tema repleto de polêmicas e discordâncias. Alternativas mais democráticas e justas para equacionar o problema do reduzido número de vagas nos cursos das universidades públicas e o elevado número de concorrentes que as disputam têm sido propostas.

Uma delas é o vestibular seriado que, ao invés de avaliar todo o conteúdo do ensino médio em apenas um exame vestibular como acontece tradicionalmente, o dilui em três avaliações anuais. Essa abertura só foi possível após a publicação da Lei n. 9.394/96 de Diretrizes e Bases da Educação Nacional LDB -, na qual está estabelecido que "a Educação Superior abrange cursos e programas de graduação, abertos a candidatos que tenham concluído o ensino médio ou equivalente e tenham sido classificados em processo seletivo" (Brasil, 1996, cap. IV, art. 44). Até a publicação do Decreto n. 99.940/90 e da Lei n. 9.934/96 a única via de acesso ao ensino superior era o vestibular tradicional.

Discussões com o objetivo de propor alternativas ao vestibular tradicional existem desde a década de 1980, sendo um de seus principais fóruns os seminários chamados Vestibular Hoje, promovidos pelo Ministério da Educação e Cultura - MEC - e algumas universidades e instituições de ensino superior. Nessas reuniões buscava-se encontrar uma modalidade de processo de seleção alternativo, que avaliasse os alunos ao longo do então $2^{\circ}$ grau, hoje ensino médio (Schilichting, Soares, Bianchetti, 2004, p. I | 6).

A primeira experiência de vestibular seriado no Brasil ocorreu no período de 1992 a 1995 com o Sistema de Avaliação Progressiva para Ingresso no Ensino Superior - Sapiens -, promovido pela Fundação Cesgranrio. O Sapiens foi um projeto-piloto aplicado no Rio de Janeiro e para sua implementação houve a necessidade de ser expedida uma autorização especial do MEC, uma vez que a legislação educacional vigente permitia a seleção de ingresso para o ensino superior apenas pelo vestibular tradicional (Schilichting, Soares, Bianchetti, 2004, p. $\mid$ |6).

objetivo primordial do Sapiens era substituir o vestibular tradicional por um conjunto de avaliações progressivas, feitas ao longo das três séries 
do ensino médio, constituindo, portanto, uma nova alternativa de acesso ao ensino superior, baseada na valorização do desempenho do aluno durante sua vida acadêmica. Esse sistema envolvia seis momentos de avaliação da aprendizagem, incluindo, além de provas objetivas e discursivas das várias disciplinas integrantes do currículo mínimo, a elaboração de redações e a aplicação de testes de aptidão verbal, numérica e abstrata. Dessa forma, o aluno era avaliado sob múltiplos aspectos e dimensões. Não foram encontrados registros sobre os resultados do Sapiens.

Em 1995, a Universidade Federal de Santa Maria - UFSM - foi pioneira ao implantar uma modalidade seriada como alternativa ao vestibular tradicional: o Programa de Ingresso ao Ensino Superior - Peies -, que atualmente seleciona $20 \%$ dos alunos de cada curso de graduação oferecido pela instituição. Ainda em 1995, a Universidade de Brasília - UnB - também discutia a possibilidade de adotar um sistema alternativo de seleção para o ingresso no ensino superior e, em 1996, introduziu o Programa de Avaliação Seriada - PAS. Responsável pelo preenchimento de $50 \%$ das vagas oferecidas pela UnB, o PAS talvez seja o vestibular seriado de maior divulgação no país, segundo o Centro de Seleção e de Promoção de Eventos - Cespe - da UnB.

Apesar de poder ser considerado um fenômeno recente na história do ingresso do ensino superior do Brasil, o vestibular seriado é uma realidade em 22 universidades públicas como modo alternativo de seleção para o ingresso no ensino superior. Em 2008, a Universidade de São Paulo - USP - lançou o PAS/USP em convênio com o Estado de São Paulo. Esse exame foi proposto com o objetivo de somar bonificações à nota final dos alunos oriundos da rede pública e foi direcionado inicialmente apenas aos alunos do $3^{\circ}$ ano do ensino médio .

A fase de maior expansão dessa modalidade de vestibular ocorreu entre os anos de 1998 e 2000, mas o número de instituições que recorrem a esse tipo de avaliação sistemática gradual pode aumentar com a aprovação do Projeto de Lei do Senado n. I | 6/06, de iniciativa do senador Cristovam Buarque. Esse documento visa alterar o art. 5 I da LDB, instituindo programas de avaliação seriada anual nos processos seletivos das universidades públicas, de modo que $50 \%$ das vagas ofertadas sejam preenchidas por essa modalidade de seleção.

O projeto de lei ainda está tramitando no Senado, com parecer favorável de seu relator. Entretanto, é possível encontrar pareceres contrários à proposta, 
como o apresentado pelo senador Paulo Paim, que critica o vestibular seriado por reforçar o caráter propedêutico do ensino médio, além de dificultar o acesso daqueles que já concluíram esse estágio da educação. Os dois estudos sobre o assunto (Schilichting, Soares, Bianchetti, 2004; Borges, Carnielli, 2005) discordam em suas conclusões. O primeiro conclui que a utilização desse processo alternativo aumenta, de fato, o ingresso de alunos oriundos de escola pública, enquanto o segundo conclui que, na verdade, o vestibular seriado é apenas uma nova forma de privilegiar o acesso ao ensino superior às camadas mais abastadas da sociedade.

Este trabalho buscou caracterizar os objetivos e estilos de avaliação de cada um desses programas e analisar de que forma os conteúdos da disciplina Biologia estão distribuídos ao longo dos módulos correspondentes às três séries do ensino médio, a fim de verificar se há consenso com relação à abordagem sequencial nessa disciplina.

\section{MATERIAIS E MÉTODOS}

A caracterização de cada programa de avaliação seriada foi feita com base nos editais disponibilizados nos sites oficiais de cada instituição, em janeiro de 2008. Os programas analisados e seus respectivos nomes, ano de início, número de vagas oferecidas e unidade federativa estão listados nos quadros I e 2.

Dentre esses vestibulares, há os que adotam o sistema de lista única, em que os candidatos são classificados por pontos decrescentes entre concorrentes de ambos os programas de vestibular, seriado e tradicional, sem reservas de vagas para cada processo. Os demais fazem listas separadas de classificação para os candidatos do vestibular seriado e do tradicional para preencher de forma independente as vagas reservadas para cada processo.

A análise comparativa entre os programas foi feita com base nos seguintes critérios: a. se o(s) objetivo(s) do programa era(m) informado(s) e qual o seu teor; b. qual a estrutura das avaliações, verificada por meio da análise dos estilos de pergunta; c. como os conteúdos da disciplina Biologia estão distribuídos ao longo de cada uma das etapas de avaliação. 


\section{QUADRO I}

\section{PROGRAMAS DE VESTIBULAR SERIADO ATUALMENTE EM VIGÊNCIA NAS REGIÕES NORTE, NORDESTE E CENTRO-OESTE DO PAÍS}

\begin{tabular}{|c|c|c|c|c|}
\hline UNIVERSIDADE & NOME DO PROGRAMA & INÍCIO & VAGAS & U.F \\
\hline $\begin{array}{l}\text { Universidade Federal de Alagoas } \\
\text {-Ufal }\end{array}$ & Processo Seletivo Seriado - PSS & 2002 & Lista única & $\mathrm{AL}$ \\
\hline $\begin{array}{l}\text { Universidade Federal do Amazo- } \\
\text { nas - Ufam }\end{array}$ & Processo Seletivo Contínuo - PSC & 1998 & $\begin{array}{r}40 \% \text { Manaus } 50 \% \\
\text { fora de Manaus } \\
\end{array}$ & AM \\
\hline $\begin{array}{l}\text { Universidade do Estado do } \\
\text { Amazonas - Ueam }\end{array}$ & $\begin{array}{c}\text { Sistema de Avaliação para Acesso ao } \\
\text { Ensino Superior - Saes }\end{array}$ & 2005 & Não divulgado & AM \\
\hline Universidade de Brasília - UnB & Programa de Avaliação Seriada - PAS & 1996 & $50 \%$ & DF \\
\hline $\begin{array}{l}\text { Universidade Estadual de Goiás } \\
\text { - UEG }\end{array}$ & Sistema de Avaliação Seriado - SAS & 2005 & $20 \%$ & GO \\
\hline $\begin{array}{l}\text { Universidade Federal do Mara- } \\
\text { nhão - UFMA }\end{array}$ & Processo Seletivo Gradual - PSG & 2001 & $50 \%$ & MA \\
\hline $\begin{array}{l}\text { Universidade do Estado do Pará } \\
\text { - UEPA }\end{array}$ & Programa de Ingresso Seriado - PRISE & 1997 & $50 \%$ & PA \\
\hline $\begin{array}{l}\text { Universidade Federal do Pará } \\
\text { - UFPA }\end{array}$ & Programa Seletivo Seriado - PSS & 2004 & Lista única & PA \\
\hline $\begin{array}{l}\text { Universidade Federal da Paraíba } \\
\text { - UFPB }\end{array}$ & Processo Seletivo Seriado - PSS & 1999 & Lista única & PB \\
\hline $\begin{array}{l}\text { Universidade Federal de Campina } \\
\text { Grande - UFCG }\end{array}$ & $\begin{array}{c}\text { Vestibular } \\
\text { modalidade seriada apenas. }\end{array}$ & 2002 & Lista única & PB \\
\hline $\begin{array}{l}\text { Universidade de Pernambuco } \\
\text { - UPE }\end{array}$ & Sistema Seriado de Avaliação - SSA & 2008 & $20 \%$ & PE \\
\hline $\begin{array}{l}\text { Universidade Federal de Roraima } \\
\text { - UFRR }\end{array}$ & Sistema Seriado - Seriado & 2007 & Não de nido & $\mathrm{RR}$ \\
\hline $\begin{array}{l}\text { Universidade Federal de Sergipe } \\
\text { - UFS }\end{array}$ & Processo Seletivo Seriado - PSS & 2002 & Lista única & SE \\
\hline
\end{tabular}




\section{QUADRO 2}

\section{DISCRIMINAÇÃO DOS PROGRAMAS DE VESTIBULAR SERIADO ATUALMENTE EM VIGÊNCIA NAS REGIÕES SUL E SUDESTE DO PAÍS}

\begin{tabular}{|c|c|c|c|c|}
\hline UNIVERSIDADE & NOME DO PROGRAMA & INÍCIO & VAGAS & U.F \\
\hline $\begin{array}{l}\text { Universidade Federal de Viçosa } \\
\text { - UFV }\end{array}$ & $\begin{array}{l}\text { Programa de Avaliação Seriada para } \\
\text { o Ingresso no Ensino Superior - Pases }\end{array}$ & 2001 & Lista única & $M G$ \\
\hline $\begin{array}{l}\text { Universidade Federal de Lavras } \\
\text { - Ufla }\end{array}$ & $\begin{array}{c}\text { Processo Seletivo de Avaliação } \\
\text { Seriada - PAS }\end{array}$ & 2000 & $40 \%$ & $M G$ \\
\hline $\begin{array}{l}\text { Universidade Federal de Juiz de } \\
\text { Fora - UFJF }\end{array}$ & Programa de Ingresso Misto - Pism & 2001 & $30 \%$ & MG \\
\hline $\begin{array}{l}\text { Universidade Federal de } \\
\text { Uberlândia - UFU }\end{array}$ & $\begin{array}{c}\text { Programa Alternativo de Ingresso ao } \\
\text { Ensino Superior - Paies }\end{array}$ & 1997 & $\begin{array}{r}50 \% \text { em cursos } \\
\text { semestrais e } 25 \% \\
\text { em anuais }\end{array}$ & $M G$ \\
\hline $\begin{array}{l}\text { Universidade Estadual de Montes } \\
\text { Claros - UniMontes }\end{array}$ & $\begin{array}{l}\text { Programa de Avaliação Seriada para } \\
\text { Acesso ao Ensino Superior - Paes }\end{array}$ & 1998 & $40 \%$ & MG \\
\hline $\begin{array}{l}\text { Universidade Federal do Vale do } \\
\text { Jequitinhonha e Mucuri - UFVJM }\end{array}$ & $\begin{array}{c}\text { Processo Seletivo de Avaliação } \\
\text { Seriada - Sasi }\end{array}$ & 2005 & $20 \%$ & MG \\
\hline $\begin{array}{l}\text { Universidade Estadual de Ponta } \\
\text { Grossa - UEPG }\end{array}$ & Processo Seletivo Seriado - PSS & 2001 & $25 \%$ & $P R$ \\
\hline $\begin{array}{l}\text { Universidade Federal de Santa } \\
\text { Maria - UFSM }\end{array}$ & $\begin{array}{c}\text { Programa de Ingresso ao Ensino } \\
\text { Superior - Peies }\end{array}$ & 1995 & $20 \%$ & RS \\
\hline $\begin{array}{l}\text { Universidade Federal de Pelotas } \\
\text { - UFPel }\end{array}$ & $\begin{array}{l}\text { Programa de Avaliação da Vida } \\
\text { Escolar - Pave }\end{array}$ & 2004 & $\begin{array}{r}\text { proporcional à } \\
\text { inscrição }\end{array}$ & RS \\
\hline
\end{tabular}

\section{RESULTADOS E DISCUSSÃO}

\section{Objetivos dos programas}

Entre as universidades pesquisadas, cinco (UFRR, UFVJM, Ueam, UFL e UFPb) não apresentaram os objetivos de seus programas nos documentos on-line. Das instituições que publicaram seus objetivos, I2 (Ufal, UnB, UEG, UFMA, UFJF, UFU, Unimontes, UFPA, UFPG, UPE, UFPel e Uepa) se referem à promulgação da LDB (Brasil, 1996), que permitiu a possibilidade de processos alternativos e, neste caso, progressivos, para o ingresso no ensino superior.

Outros objetivos citados em nove dessas instituições se referem à possibilidade de autoavaliação pelo aluno e de correções de suas deficiên- 
cias, sendo que em sete (UnB, UFMA, UFV, UFJF, UFPel, Uepa e UFSM) é manifestada também a preocupação de propiciar maior integração entre universidade e escola. Nos programas da UFU e UFPA o objetivo é estimular o aluno.

\section{Estrutura das avaliações}

Todas as universidades, exceto a UFCG, possuem vestibular seriado e vestibular tradicional realizados de modo concomitante. Com isso, o aluno do terceiro ano do ensino médio pode inscrever-se nas duas seleções e, desta forma, ter sua chance de ingresso aumentada. Essa, aliás, é uma das justificativas apresentadas pela reitoria da UFMA para a extinção progressiva do seu Programa de Vestibular Seriado - PSG - a partir de 2007.

Com exceção da Universidade Federal de Campina Grande, cuja estrutura de avaliação é desenvolvida em dois anos, todas as outras instituições apresentam seu vestibular seriado em programas trienais. Com essa configuração, o aluno pleiteante a uma vaga na universidade realiza uma avaliação ao final de cada ano letivo. Na UFCG, a primeira avaliação concentra as matérias relativas ao primeiro e segundo ano do ensino médio, enquanto a segunda avaliação é relativa apenas ao conteúdo programático do terceiro ano.

Na UFSM, UFG e na UFL há uma segunda fase para os que concluíram as três avaliações, sendo que na UFSM e na UFG os alunos são selecionados por meio da pontuação obtida no conjunto das provas aplicadas nos três anos e classificados em ordem decrescente. A nota de corte é estabelecida com base na proporção de três candidatos/vaga em Goiás e de quatro candidatos/vaga em Santa Maria.

Na Ufam, Ufal, UFU, Unimontes, UFPel, UFRR, Uepa e UFPG, as provas do processo seletivo seriado são aplicadas em um único dia. A UVJM também organiza as avaliações em um único dia porém o $3^{\circ}$ módulo é realizado em dois períodos (manhã e tarde). Na UFL, UFCG e UFV, as provas dos três módulos são divididas em dois dias diferentes. A separação das matérias relativas a cada um dos dias é específica de cada universidade. Já a UEG, UFMA, UnB, Uepa, UFPB, UFS e UFSM organizam as provas do primeiro e segundo módulo em um dia, e as do $3^{\circ}$ módulo em dois dias. A UFJF tem o calendário 
mais atípico de aplicação de provas: os três módulos são realizados em quatro dias diferentes, cada um referente a duas disciplinas do programa. A UPE e a UEA não divulgaram essa informação.

Apesar das peculiaridades inerentes a cada programa, pudemos identificar três diferentes grupos de estilos de avaliação:

I. Provas de conhecimentos gerais nos três anos, e no terceiro somase uma atividade de redação. Nessas avaliações de conhecimentos gerais, podemos encontrar universidades que se utilizam de:

a. perguntas objetivas apenas (Ufam, Unimontes, UFS, UEG, Uepa, UFRR e UFPel);

b. perguntas objetivas e dissertativas na mesma prova (Ufal e UnB);

c. perguntas objetivas nos dois primeiros anos e objetivas e dissertativas apenas no terceiro ano (UFMA, UFPA, UFPb e UFVJM);

2. Provas de conhecimentos gerais com questões objetivas e/ou dissertativas e redação nas três fases (UFV, UFU, UFPG, UFL e UFSM).

3. Provas de conhecimentos gerais nas três fases com questões objetivas e dissertativas, mas que não incluem redação (UFJF e UFCG).

Considerou-se perguntas objetivas as de múltipla escolha com quatro ou cinco alternativas, as que apresentam soma de valores, ou ainda as que trabalham com proposições verdadeiras e falsas. A UPE e a Ueam não divulgaram essa informação.

\section{Conteúdos de Biologia}

A distribuição dos conteúdos curriculares de Biologia ao longo dos três anos do vestibular seriado nas instituições em análise está sumariada na figura I. É possível notar neste gráfico que no primeiro ano há prevalência dos temas Citologia ( 7 referências) e Histologia (14); no segundo ano de tópicos de Seres vivos ( 14 ) e suas características fisiológicas ( 14 ); e no terceiro ano, de Genética ( I3), Ecologia ( 12 ) e Evolução ( 15 ). 
GRÁFICO DA FREQUÊNCIA ABSOLUTA DA DISTRIBUIÇÃO DE CONTEÚDOS DE BIOLOGIA EXIGIDOS PELAS UNIVERSIDADES QUE UTILIZAM O VESTIBULAR SERIADO EM CADA UM DOS TRÊS ANOS DO PROCESSO

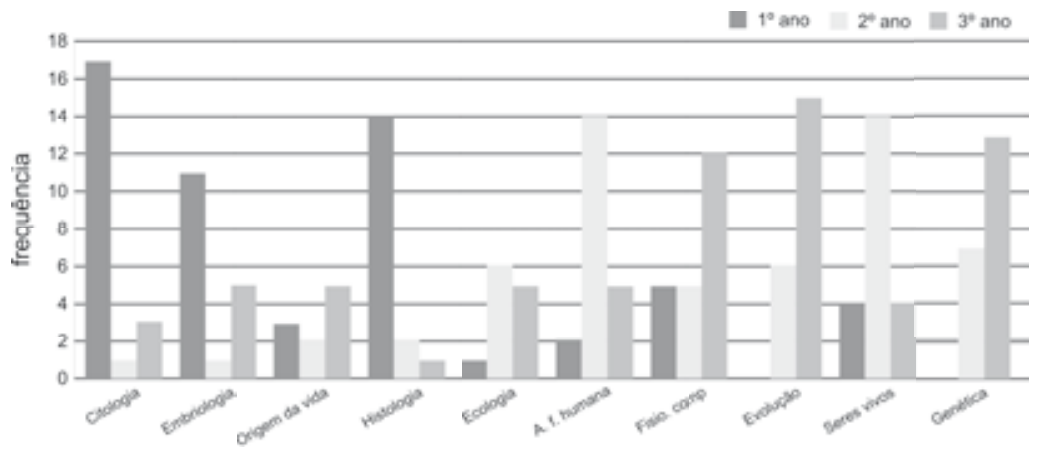

Abreviações: A. F. = Anatomia e Fisiologia; Fisio. Comp. = Fisiologia Comparada.

Os demais assuntos aparecem de maneira mais dispersa ao longo dos três anos, com exceção de Evolução e Genética que não são exigidos no primeiro módulo em nenhum dos vestibulares.

O estado de Minas Gerais é o que concentra o maior número de universidades que utilizam o processo seletivo seriado como forma de ingresso para seus cursos superiores. São seis instituições, como resumido no quadro 3.

\section{QUADRO 3}

CONTEÚDOS DE BIOLOGIA EXIGIDOS EM CADA UM DOS TRÊS ANOS NOS VESTIBULARES SERIADOS DAS UNIVERSIDADES DO ESTADO DE MINAS GERAIS

\begin{tabular}{c|c|c|c}
\hline & $1^{\circ}$ ano & $2^{\circ}$ ano & $3^{\circ}$ ano \\
\hline UFJF & Citologia e Histologia & $\begin{array}{c}\text { Seres Vivos, Fisiol. Comp. } \\
\text { e Ecologia }\end{array}$ & $\begin{array}{c}\text { Evolução, Genética, Ecologia } \\
\text { e Embriologia }\end{array}$ \\
\hline Unimontes & Citologia e Ecologia & $\begin{array}{c}\text { Seres Vivos, Genética e } \\
\text { Evolução }\end{array}$ & $\begin{array}{c}\text { Histologia, Fisiol. Comp. e } \\
\text { Embriologia }\end{array}$ \\
\hline UFU & $\begin{array}{c}\text { Histologia, Ecologia e } \\
\text { Embriologia }\end{array}$ & $\begin{array}{c}\text { Seres Vivos, Fisiol. Comp. } \\
\text { e Anat. e Fisiol. Humana }\end{array}$ & $\begin{array}{c}\text { Citologia, Genética, Origem } \\
\text { da Vida e Evolução }\end{array}$ \\
\hline UFVJM & $\begin{array}{c}\text { Citologia e Histologia } \\
\text { UFV/Ufla }\end{array}$ & $\begin{array}{c}\text { Seres Vivos e Fisiol. } \\
\text { Comp. }\end{array}$ & $\begin{array}{c}\text { Genética, Evolução e } \\
\text { Ecologia }\end{array}$ \\
\hline
\end{tabular}

Obs.: As áreas sombreadas evidenciam as diferenças entre os conteúdos dos módulos. 


\section{CONCLUSÃO}

Os diferentes programas de vestibular seriado não apresentam uniformidade em diversos aspectos, como número de vagas, estrutura de avaliação e conteúdo programático. As variações observadas nas sequências de conteúdos nas diferentes unidades federativas e, dentro de um mesmo estado, como ocorre em Minas Gerais, evidenciam que há pouca concordância sobre o encadeamento ideal para o ensino de Biologia. Os vestibulares seriados que estabelecem uma ordem de saberes para cada ano do ensino médio podem vir a interferir na programação das escolas de cada região, trazendo como primeira consequência a restrição para que o aluno possa se preparar para as provas em mais de uma instituição. A adoção quase compulsória de um programa preestabelecido como norteador da estrutura curricular de uma escola deve ser discutida, uma vez que os professores podem sentir-se tolhidos em suas decisões com relação ao que consideram adequado aos seus alunos, interferindo na autonomia pedagógica assegurada pelo art. 26, da LDB/96, e valorizada pelos Parâmetros Curriculares Nacionais do Ensino Médio.

Outro aspecto que merece atenção é a possibilidade de reforçar o caráter propedêutico do ensino médio, correndo-se o risco de transformar cada uma das três séries em um cursinho preparatório para o vestibular, desvirtuando o objetivo maior desse nível de escolaridade que é o aprimoramento do educando como ser humano, sua formação ética, autonomia intelectual e pensamento crítico, preparação para o mundo do trabalho e o desenvolvimento de competências para continuar seu aprendizado (Brasil, 1996, art. 35).

O vestibular seriado, apesar de ser uma alternativa aparentemente menos traumática para o aluno em razão do menor conteúdo em cada avaliação, ainda precisa ser amplamente discutido; a necessidade de homogeneidade de currículo deve ser ponderada considerando, a enorme diversidade cultural do país. Da maneira como está, ca a dúvida se o vestibular seriado é a solução que se deve adotar para resolver ou minimizar o problema do ingresso no ensino superior.

\section{REFERÊNCIAS BIBLIOGRÁFICAS}

BRASIL. Decreto n.99.490, de 30 agosto de 1990: delega às instituições de ensino superior a competência para realização do concurso vestibular, nos termos da lei, de seus estatutos e regimentos. Diário Oficial da União, Brasília, D.F., p. 16.612, 31 ago. 1990. 
Lei n. 9.394, de 23 dez. 1996: estabelece as diretrizes e bases da educação nacional. Diário Oficial da União, Brasília, p.27.833, 23 dez.1996.

Parâmetros Curriculares Nacionais para o Ensino Médio - PCNEM. Disponível em: http://portal.mec.gov.br/seb/index.php?option=content\&task=view \&id $=265 \&$ ltemid $=255$. Acesso em: 17 jan. 2008.

Projeto de lei do Senado n. I /6/2006. Disponível em:http://www.senado. gov.br/sf/atividade/Materia/getHTML.asp?t=8056. Acesso em: 17 jan 2008.

BORGES, J. L. C.; CARNIELLI, B. L. Educação e estrati cação social no acesso à universidade pública. Cadernos de Pesquisa, São Paulo, v.35, n. I24, p. I13-139, jan./abr. 2005.

SCHILICHTING, A. M. S., SOARES, D. H. P.; BIANCHETTI, L. Vestibular seriado: análise de uma experiência em Santa Catarina. Psicologia \& Sociedade, Santa Catarina, n. I6, v.2, p. I |4- | 26, maio/ago.2004.

\section{INSTITUIÇÕES}

CESGRANRIO. Sistema de Avaliação Progressiva para Ingresso no Ensino Superior - Sapiens (1992 a 1995). Disponível em: http://www.cesgranrio.org.br/eventos/avaliacoes/avaliacoes_2. html. Acesso em: 16 jan. 2008.

UnB. Cespe. Disponível em: http://www.cespe.unb.br/pas/guiapas2007/sistema_unb_de_ avaliacao_e_selecao.htm. Acesso em: 16 jan 2008.

UEA. Disponível em: http://www4.uea.edu.br/?dest=saes . Acesso em: 25 jan. 2008.

UEG. Disponível em: http://www.sas.ueg.br/. Acesso em: 6 jan. 2008.

UEPA. Disponível em: http://www2.uepa.br/seletivo2007/ ; http://www2.uepa.br/seletivo2007/prise/doc/prise\%20-\%20edital\%20e\%20subprogramaXI.pdf. Acesso em: 6 jan. 2008.

UEPG. Disponível em: http://www.uepg.br/pss/Documentos/2007/EDTI 2-2007-PSS.pdf; http://www.uepg.br/pss/Documentos/2007/EDT I 3-2007-PSS.pdf; http://www.uepg.br/pss/ Documentos/2007/EDTI I-2007-PSS.pdf. Acesso em: 6 jan. 2008.

UFAL. Comissão Permanente do Vestibular - Copeve. Disponível em: http://www.copeve. ufal.br/sistema/pss/Processo\%20Seletivo\%20Uni cado\%202008/Manual\%20PSS\%202008. pdf. Acesso em: 6 jan. 2008.

UFAM. Comissão Permanente de Concursos - Comvest. Disponível em: http://www. comvest.ufam.edu.br/arquivo/resolucao_psc.pdf. Acesso em 6 jan. 2008. 
UFCG. Comprov. Disponível em: http://www.comprov.ufcg.edu.br/programa05.pdf; http:// www.comprov.ufcg.edu.br/provas06.htm; http://www.comprov.ufcg.edu.br/programa05. pdf. Acesso em: 6 jan. 2008.

UFJF. Disponível em: http://www.vestibular.ufff.br/; http://www2.vestibular.ufjf.br/ index. php? module $=$ vestibular\&action=html: les:geral2008:programas.pdf. Acesso em: 6 jan. 2008.

UFLA. Comissão de Processo Seletivo - Copese. Disponível em: http://www.copese.ufla. br/copese/upload/File/2008_I/PAS/2A_ETAPA/EDITAL_PAS2.pdf; http://www.copese.ufla.br/ copese/upload/File/2008_I/PAS/IA_ETAPA/ProgramaPASI etapa_dez2007.pdf; http://www. copese.ufla.br/copese/upload/File/2008_I/PAS/2A_ETAPA/ProgramaPAS2etapa_dez2007. pdf; http://www.copese.ufla.br/copese/upload/File/2008_I/PAS/3A_ETAPA/ProgramaPAS3etapa_dez2007.pdf. Acesso em: 6 jan. 2008.

UFMA. Disponível em: http://www.old.ufma.br/vestibular/PSG\%20III\%20(20042006)/EDITAL/Edital_PSG_2004_2006_III.pdf; http://www.old.ufma.br/vestibular/ PSG\%201I\%20(2005-2007)/PROGRAMA_DISCIPLINAS/ProgramasDisciplinasPSG22005-2007.pdf. Acesso em: 6 jan. 2008.

UFPA. Departamento de Apoio ao Vestibular - Daves. Disponível em: http://www.daves. ufpa.br/pss2007/MANUAL\%20PSS\%202007\%20CANDIDATO.pdf; http://www.daves.u fpa.br/pss2007/conteudo\%20programatico/6-Programa\%20de\%20Biologia\%20PSS-2007. pdf. Acesso em: 6 jan. 2008.

UFPB. Comissão Permanente do Concurso Vestibular - Coperve. Disponível em: http://hww. coperve.ufpb.br/ProgramasDasMaterias.pdf. Acesso em: 6 jan. 2008.

UFPel. Disponível em: http://vestibular.ufpel.edu.br/pave/; http://vestibular.ufpel.edu.br/ pave/download/edital_pave_etl.pdf; http://vestibular.ufpel.edu.br/pave/download/programa_pave_2_etapa.pdf. Acesso em: 24 jan. 2008.

UFRR Comissão Permanente de Vestibular - CPV. Disponível em: http://www.ufrr.br/vestibular/; http://www.ufrr.br/noticias/vestibular/vestibular-seriado-interessados-devem-fazerinscricao-ate-sexta. Acesso em: 24 jan. 2008.

UFS. Manual do candidato. Disponível em: http://www.vestibular2008.ufs.br/arquivos2008/ Manual2008.pdf. Acesso em: 6 jan. 2008.

UFSM. Comissão Permante do Vestibular - Coperves. Disponível em: http://w3.ufsm.br/ coperves/peies/; http://coralx.ufsm.br/coperves/not.php?id_noticia=19. Acesso em: 30 ago. 2007. 
UFU. Disponível em: http://mww.ingresso.ufu.br/Paies2008/pdf/Manual_IEtapa_2007-20 I 0. pdf; http://www.ingresso.ufu.br/Paies2008/pdf/Manual_2etapa_2006_2009.pdf; http://www. ingresso.ufu.br/Paies2008/pdf/Manual_3etapa_2005-2008.pdf. Acesso em: 6 jan. 2008.

UFV.Disponível em: http://www.processoseletivo.ufv.br/sisvest_concursos/scripts/PAS2007I/; http://www.processoseletivo.ufv.br/sisvest_concursos/scripts/PAS2006-2/; http://www. processoseletivo.ufv.br/sisvest_concursos/ les/PAS2005-3/manual_pas2005_3.pdf. Acesso em: 6 jan. 2008.

UFVJM. Disponível em: http://www.ufvjm.edu.br/copese/vestibular/sasi_2007/. Acesso em: 24 jan. 2008.

UNIMONTES. Comissão Técnica de Concursos - Cotec.Disponível em: http://www.cotec. unimontes.br/paes/paes2007/ManualCandidatoPAES_2007.pdf; http://www.cotec.unimontes. br/paes/paes_informativo.htm. Acesso em: 6 jan. 2008.

UPE. Disponível em: http://www.upenet.com.br/. Acesso em: 24 jan. 2008.

Recebido em: julho 2008

Aprovado para publicação em: dezembro 2009 